\section{FLARES IN PATIENTS WITH SYSTEMIC LUPUS ERYTHEMATOSUS}

${ }^{1} \mathrm{~K}$ McElhone, ${ }^{2} \mathrm{~J}$ Abbott, ${ }^{3} \mathrm{M}$ Hurley, ${ }^{4} \mathrm{P}$ Lanyon, ${ }^{5} \mathrm{~A}$ Rahman, CS Yee ${ }^{6},{ }^{7} \mathrm{M}$ Akil, ${ }^{8} \mathrm{Y}$ Ahmad, ${ }^{9}$ B Bruce, ${ }^{10} \mathrm{C}$ Gordon, ${ }^{1} \mathrm{LS}$ Teh*. ${ }^{1}$ Royal Blackburn Hospital, Rheumatology, Blackburn, UK; ${ }^{2}$ University of Central Lancashire, School of Psychology, Preston, UK; ${ }^{3}$ University of Central Lancashire, College of Health and Wellbeing, Preston, UK; ${ }^{4}$ Nottingham University Hospitals, Rheumatology, Nottingham, UK; ${ }^{5}$ University College London, Centre of Rheumatology Research, London, UK; ${ }^{6}$ Doncaster Royal Infirmary, Rheumatology, Doncaster, UK; ${ }^{7}$ Royal Hallamshire Hospital, Rheumatology, Sheffield, UK; ${ }^{8}$ Llandudno Hospital, Peter Maddison Rheumatology Centre, Llandudno, UK; ${ }^{9}$ University of Manchester, The Kellgren Centre for Rheumatology, Manchester, UK; ${ }^{10}$ University of Birmingham, Rheumatology Research Group, Birmingham, UK

10.1136/lupus-2017-000215.460
Background and aims Systemic lupus erythematosus (SLE) is characterised by relapses and remissions. This study describes the frequency, type and time to flare in a cohort of SLE patients.

Methods SLE patients with one or more "A" or "B" BILAG2004 systems meeting flare criteria (items that were "new" or "worse") and requiring an increase in immunosuppressive therapy were recruited from nine UK centres and assessed at baseline and monthly for nine months. Flares were defined as: severe (" $\mathrm{A}$ " flare/s irrespective of number of "B" flares), moderate ( 2 or more "B" flares without any " $\mathrm{A}$ " flares and mild (one "B" flare).

Results Of the 100 patients, 94\% were female, 61\% white Caucasians, mean age (SD) was 40.7 years (12.7) and mean

Abstract 460 Table 1 Type and frequency of Flares.

\begin{tabular}{|c|c|}
\hline $\begin{array}{l}\text { Types of Flares (using BILAG-2004 index) over ninemonths per } \\
\text { patient ( } n=100 \text { ) }\end{array}$ & Number of patients $(n)$ \\
\hline \multicolumn{2}{|l|}{ Severe (Any As irrespective of Bs) } \\
\hline Any severe flare & 22 \\
\hline Only one severe flare & 15 \\
\hline Multiple severeflares & 7 \\
\hline Severe flares only (without moderate/mild flares) & 7 \\
\hline Severe and Moderate Flares only & 0 \\
\hline Severe and moderate and mild flares & 3 \\
\hline Severe and Mild Flares only & 12 \\
\hline \multicolumn{2}{|l|}{ Moderate (Two or more Bs withoutany A) } \\
\hline Any moderate Flare & 19 \\
\hline Only one moderate Flare & 12 \\
\hline Multiple moderate Flare & 7 \\
\hline Moderate Flares only (without severe/mild flares) & 2 \\
\hline Moderate and Severe Flares only & 0 \\
\hline Moderate and Severeand mildflares & 3 \\
\hline Moderate and Mild filares only & 14 \\
\hline \multicolumn{2}{|l|}{ Mild (one B) } \\
\hline Any mild flare & 67 \\
\hline Only one Mild flare & 36 \\
\hline Multiple Mild flares & 31 \\
\hline Mild Flares only (without severe/moderateflares) & 38 \\
\hline Mild and Severe Flares only & 12 \\
\hline Mild and Severe and Moderate & 3 \\
\hline Mild and moderate Flares only & 14 \\
\hline \multicolumn{2}{|l|}{ No Flares (no A or B scores) } \\
\hline Patients with no $A$ or $B$ scores & 24 \\
\hline
\end{tabular}


Time to first flare

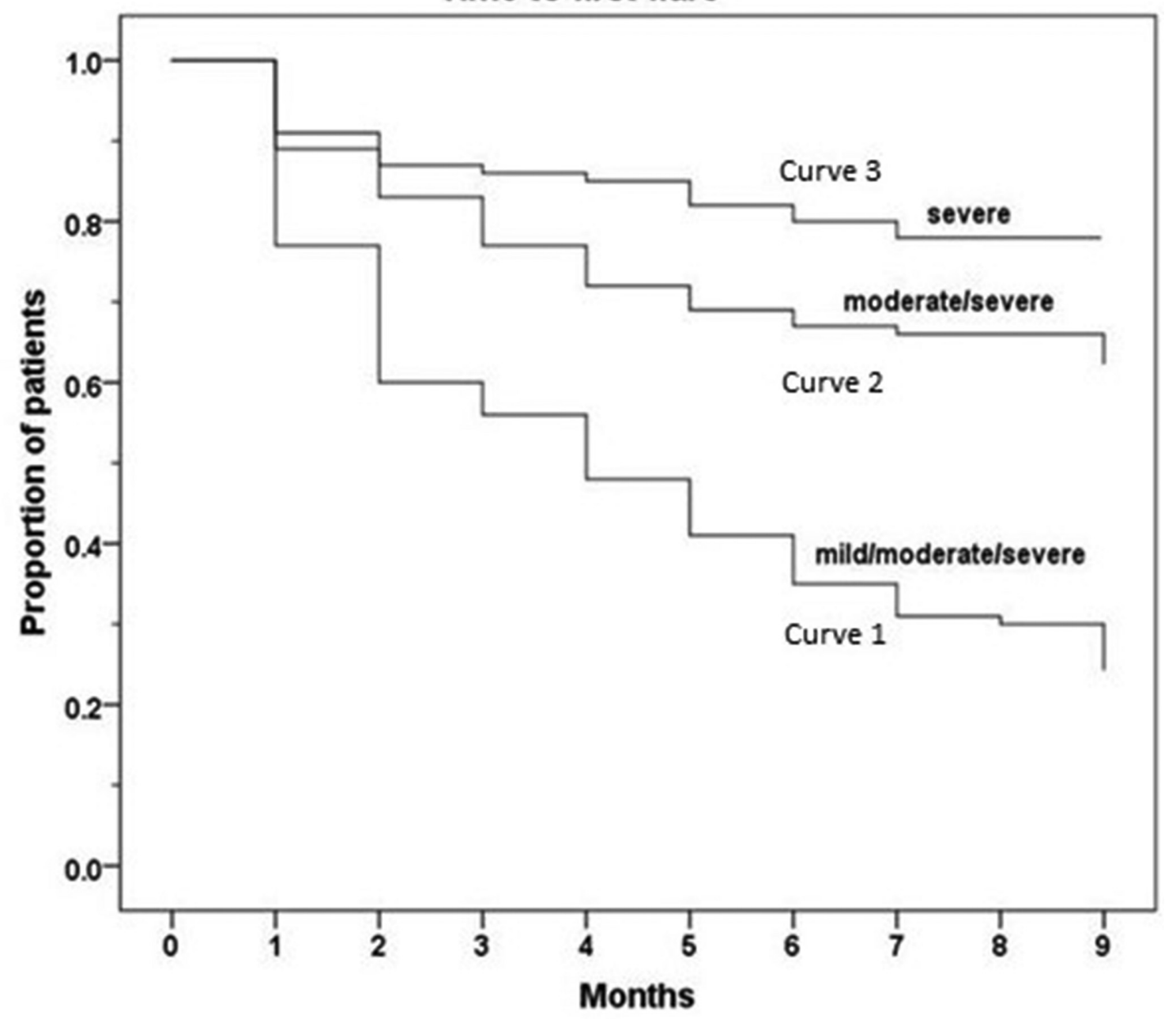

\section{Abstract 460 Figure 1}

disease duration (SD) 9.3 years (8.1). A total of 195 flares occurred in 76 patients over 781 monthly assessments, giving a flare rate of $0.25 /$ month. There were 37 severe flares (22 patients), 32 moderate flares (19 patients) and 126 mild flares (67 patients) [Table 1]. The median time to any "A" or "B" flare was 4 months (95\% CI 2.7 to 5.3 months). Figure 1 shows the time to the first mild/moderate/severe flare (Curve 1), moderate/severe flare (Curve 2) and severe flare (Curve 3). Table 2 shows that severe and moderate flares tend to be in the system/s affected at baseline whereas mild flares are more likely to affect any system.

Conclusions This real world cohort will share similarities with populations recruited to clinical studies so these results may inform future trial design.

\section{ASSESSING THE CONTENT VALIDITY OF THE SF-36, THE SKINDEX-29+3 PATIENT REPORTED OUTCOMES (PRO) INSTRUMENTS, AND PAIN, FATIGUE IN PATIENTS WITH CUTANEOUS LUPUS ERYTHEMATOSUS (CLE)}

${ }^{1} \mathrm{M}$ Vanya*, ${ }^{1} \mathrm{M}$ Cho, ${ }^{2} \mathrm{~S}$ Chen, ${ }^{2} \mathrm{~A}$ Kao, ${ }^{1} \mathrm{~K}$ Howard, ${ }^{3} \mathrm{VP}$ Werth. ${ }^{1} \mathrm{CCON}$ plc, Clinical Outcomes Assessments, San Francisco, USA; ${ }^{2}$ Biogen, HEOR, Boston, USA; ${ }^{3}$ Corporal Michael J. Crescenz Veterans Affairs, Medical Centre Philadelphia PA, Philadelphia, USA
Background and aims Patients with CLE may experience symptoms, which can negatively impact their quality of life. The objectives of this study were as follows: 1) to investigate patient experiences associated with CLE, 2) to investigate the impact of living with CLE on patients' lives, 3) to evaluate the content validity of the 36-item Short Form Health Survey (SF-36) and the Skindex-29+3 PRO instruments in the CLE population, and 4) to evaluate the appropriateness of a shorter recall period of 1 week instead of 4 weeks for the Skindex $-29+3$.

Methods This cross-sectional qualitative study utilised a combined concept elicitation (CE) and cognitive interview (CI) method. Study participants were recruited across three US clinical sites and interviewed one-on-one during a $90 \mathrm{~min}$ inperson interview. Interviews followed a semi-structured interview guide that elicited patient experiences with CLE and its impact on patients' lives, followed by CI that assessed patients' understanding and interpretation of each instrument. Results Patients found these instruments to be readily understandable, interpreting items correctly and with minimal difficulty. Patients reported a one-week recall period for the Skindex-29+3 to be meaningful and easy to use, although many stated that their symptoms and experiences were felt over a longer period of time. 